\title{
Energy coupling mechanisms of AcrB-like RND transporters
}

\author{
Xuejun C. Zhang ${ }^{1,2 凶}$, Min Liu ${ }^{1,2}$, Lei Han ${ }^{1,2}$ \\ 1 National Laboratory of Biomacromolecules, CAS Center for Excellence in Biomacromolecules, Institute of \\ Biophysics, Chinese Academy of Sciences, Beijing 100101, China \\ 2 College of Life Science, University of Chinese Academy of Sciences, Beijing 100049, China
}

Received: 4 May 2017 / Accepted: 27 July 2017 / Published online: 25 September 2017

\begin{abstract}
Prokaryotic AcrB-like proteins belong to a family of transporters of the RND superfamily, and as main contributing factor to multidrug resistance pose a tremendous threat to future human health. A unique feature of AcrB transporters is the presence of two separate domains responsible for carrying substrate and generating energy. Significant progress has been made in elucidating the three-dimensional structures of the homo-trimer complexes of AcrB-like transporters, and a three-step functional rotation was identified for this class of transporters. However, the detailed mechanisms for the transduction of the substrate binding signal, as well as the energy coupling processes between the functionally distinct domains remain to be established. Here, we propose a model for the interdomain communication in AcrB that explains how the substrate binding signal from the substrate-carrier domain triggers protonation in the transmembrane domain. Our model further provides a plausible mechanism that explains how protonation induces conformational changes in the substrate-carrier domain. We summarize the thermodynamic principles that govern the functional cycle of the AcrB trimer complex.
\end{abstract}

Keywords AcrB, RND transporter, Membrane potential

\section{INTRODUCTION}

In Gram-negative bacteria, the periplasm functions as a protective buffer zone against cytotoxic substances from the extracellular environment (Li et al. 2015). The cell actively expels toxic compounds that have penetrated the outer membrane and that have accumulated in the periplasmic space, thus preventing the toxins from either harming the cell from within the periplasm, or further penetrating into the cytosol. In addition, metabolic waste and other cytotoxic compounds transported from cytosol to periplasm should also be expelled promptly. Resistance-nodulation-cell division (RND) type of transporters plays essential roles in these

Electronic supplementary material The online version of this article (doi:10.1007/s41048-017-0042-y) contains supplementary material, which is available to authorized users.

$\triangle$ Correspondence: zhangc@ibp.ac.cn (X. C. Zhang) toxin-extrusion processes vital for cell survival (Higgins 2007).

RND transporters form a superfamily of membrane proteins that play crucial roles in diverse biological processes from multidrug resistance in bacteria to trafficking of lipid molecules in eukaryotic cells (Higgins 2007). RND proteins unidirectionally transport substrates between two distinct micro-environments not necessarily separated by a biological membrane. In particular, RND-mediated transport often occurs against the substrate chemical potential, e.g., from a low-concentration to a high-concentration environment, or from a highaffinity carrier to a low-affinity carrier. Such transport requires external energy, and RND transporters are known to utilize the electrochemical potential of protons, i.e., the proton motive force (PMF), to drive their substrate-transport processes (Yamaguchi et al. 2015).

The AcrAB-TolC tripartite complex, a prototypical member of the RND family, is constitutively expressed in 
many Gram-negative bacteria, is responsible for efflux transport of lipophilic and/or amphipathic compounds (a common property of many cytotoxic substances such as antibiotics), and thus plays important roles in multidrug resistance (Nikaido and Takatsuka 2009; Yamaguchi et al. 2015). In this complex, AcrB captures a wide variety of structurally dissimilar drugs and toxic compounds from either periplasm or the periplasmic leaflet of the inner membrane and delivers them to a conduit formed by a TolC trimer which connects to the exterior of the cell. Analysis of amino acid sequences shows that AcrB belongs to the Hydrophobe/Amphiphile Efflux-1 (HAE1) family of the RND superfamily (Perrin et al. 2010). Hereafter, we refer to members of the HAE1 family and other phylogenetically related RND families as AcrB-like transporters (Fig. S1). From the view of energy coupling, AcrB-like transporters are distinct from ATP-binding cassette $(\mathrm{ABC})$ transporters. In most cases, $\mathrm{ABC}$ transporters utilize cytosolic energy source (i.e., ATP hydrolysis) to drive cross-membrane transport of substrates. In contrast, AcrB-like transporters utilize PMF across the bacterial inner membrane to transport substrates, either within an aqueous environment or at the aqueous-membrane interface.

Structures, substrate recognition mechanisms, and the functional cycle of AcrB-like transporters have been extensively reviewed previously (Higgins 2007; Nikaido and Takatsuka 2009; Li et al. 2015; Yamaguchi et al. 2015). Here, we focus on the structural basis of the energy coupling mechanisms of AcrB-like transporters. We will first summarize the structural features of the AcrB complex for the sake of discussion of the mechanistic principles, and then propose a model on the energy coupling mechanism of this family of transporters between the substrate-carrier domain and the transmembrane (TM) PMF-driven motor domain. All arguments discussed below are based on insights from Escherichia coli AcrB (Ec-AcrB), unless stated otherwise.

\section{BASIC STRUCTURES OF ACRB}

The AcrAB-TolC tripartite complex from E. coli is the most extensively studied RND transporter (Yamaguchi et al. 2015). Recent cryo-electron microscopy studies revealed that this complex is of a stoichiometry of $\mathrm{AcrB}_{3}: \mathrm{AcrA}_{6}: \mathrm{TolC}_{3}$ (Du et al. 2014; Jeong et al. 2016). As shown schematically in Fig. 1, AcrB serves as the PMFdriven transporter and forms a homo-trimer; the TolC trimer forms a conduit connecting the exit chamber of the AcrB trimer to exterior of the cell; and the AcrA hexamer serves as an adaptor between the AcrB and TolC trimers.

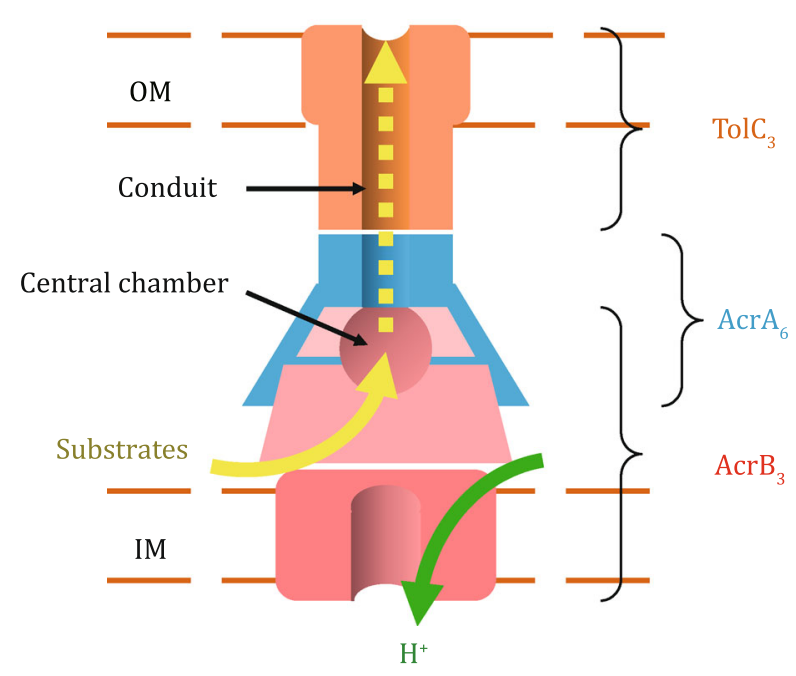

Fig. 1 Schematic diagram of the AcrAB-TolC complex. "OM" and "IM" stand for outer and inner membranes, respectively

Among the three component proteins in the E. coli AcrAB-TolC complex, AcrB (GenBank access ID: ANK05811.1), consisting of 1,049 amino acid residues, is the most extensively studied protein both structurally and functionally. Currently, the Protein Data Bank (PDB) database (Berman et al. 2000) contains over 40 fulllength AcrB crystal structures, demonstrating that the stable homo-trimer is the functional form of AcrB. Each protomer is composed of three major domains, namely the TM domain; the substrate-carrier, porter domain (formerly called port domain); and the TolC-docking domain (Murakami et al. 2002) (Fig. 2). The TM domain contains 12 TM helices (TMs 1-12) (Fig. 2C; Fig. S1A) and resides in the inner membrane with both its $\mathrm{N}$ - and $\mathrm{C}$-termini located in the cytosol. This domain assumes a pseudo twofold symmetry between the N-terminal subdomain (termed $\mathrm{N}_{\mathrm{TM}}$, composed of TMs 1-6) and C-terminal subdomain $\left(\mathrm{C}_{\mathrm{TM}}\right.$, TMs $\left.7-12\right)$, with the rotation axis perpendicular to the membrane. Around the symmetry axis, TM4 and TM10 form a parallel central helix-pair. Functionally important residues D407, D408, and K940 (numbered as in Ec-AcrB) are located in the TM4-TM10 interface (Fig. 3A) and form a rather extensive hydrogen $(\mathrm{H})$-bond network (Middlemiss and Poole 2004; Eicher et al. 2012). In particular, D407 is located in a highly conserved sequence motif, $\mathrm{GX}_{3} \mathrm{DX}_{6} \mathrm{E}$, of AcrB-like transporters (Perrin et al. 2010) (Fig. S1). Integrity of this H-bond network is essential for the transport function of AcrB-like transporters, and the D407-D408 acidic pair is identified to be the protonation site required for PMF-energy conversion $\mathrm{Cu}^{\mathrm{Mu}}$ rakami et al. 2002). Furthermore, between $\mathrm{N}_{\mathrm{TM}}$ and $\mathrm{C}_{\mathrm{TM}}$ (particularly between TMs 6 and 7), a long amphipathic helix ( $\sim 26$ residues, termed a6-7) represents a 
A

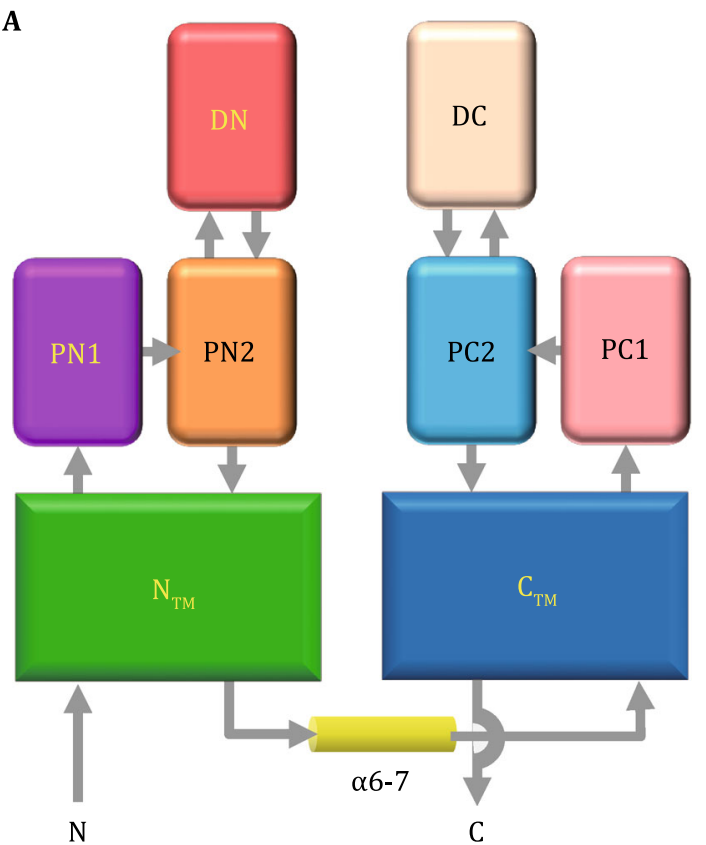

C

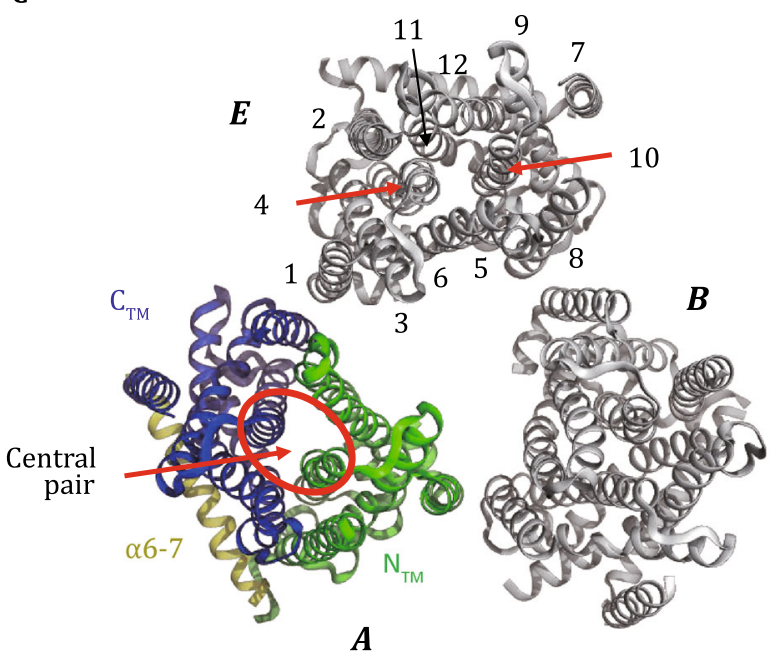

B

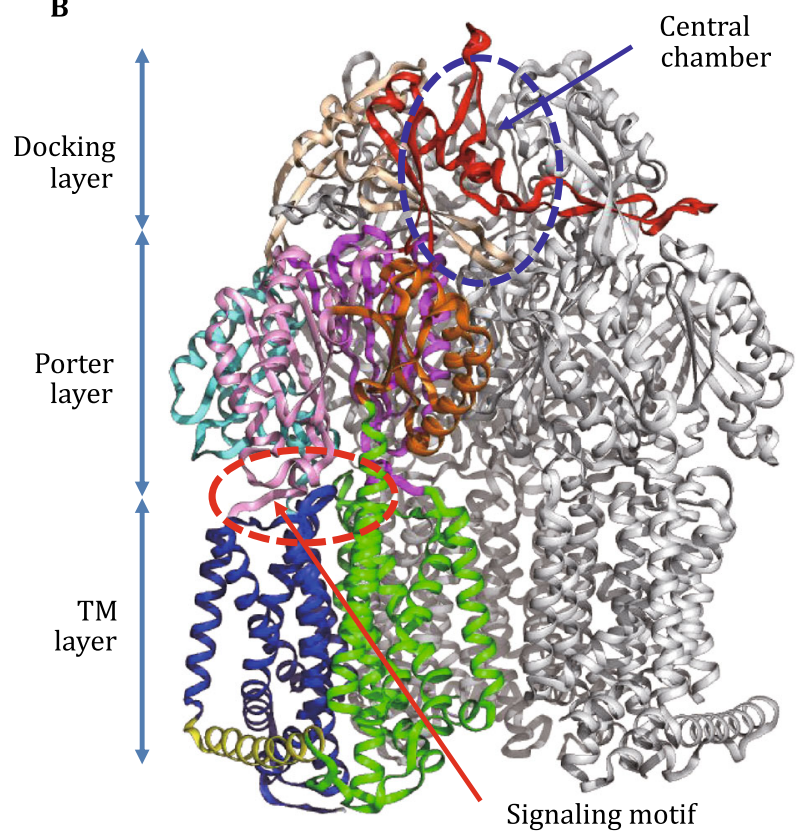

D

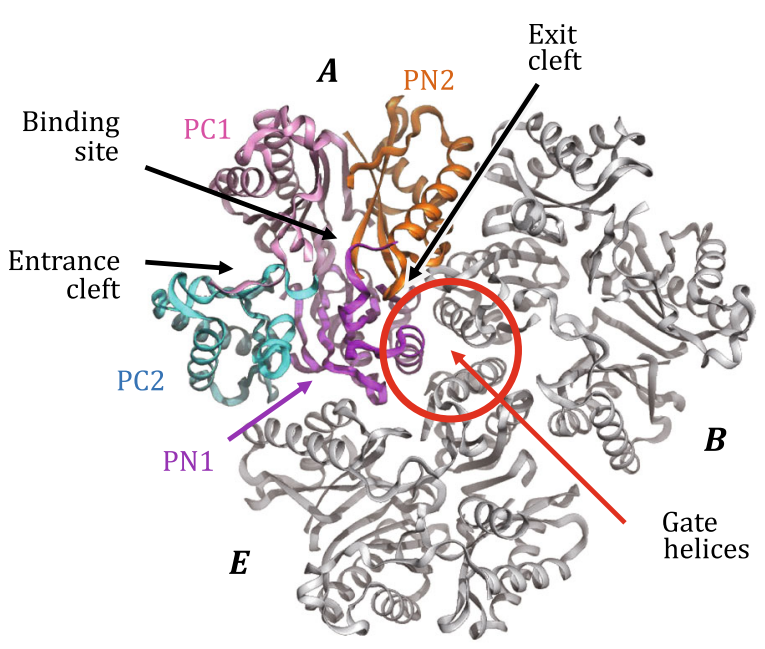

Porter layer (bottom view)

TM layer (top view)

Fig. 2 Crystal structures of the AcrB asymmetric trimer. A The topology of the AcrB protomer. B-D The AcrB trimer (PDB ID: 4DX5) is shown in ribbon diagrams. In each panel, the access protomer is shown in color, while the other two protomers are displayed in gray. In $\mathbf{C}$ and $\mathbf{D}$, the access, binding, and extrusion protomers are labeled as " $A$ ”, “ $B$ ", and “ $E$," respectively. In the top view of the TM layer (C), TM helices are labeled as " 1 " through " 12 "

conserved structural feature of RND transporters (Fig. 2) (Murakami et al. 2002; Sennhauser et al. 2009; Gong et al. 2016). It presumably lies on the cytosolic surface of the inner membrane, forming part of the rim of the AcrB trimer complex. A highly conserved tyrosine residue, Y527 (Fig. S1), anchors $\alpha 6-7$ to another conserved region at the $\mathrm{C}$-terminal end of TM12.
The porter domain contains four topologically homologous subdomains, namely PN1, PN2, PC1, and PC2, each of which consists of two $\beta-\alpha-\beta$ repeats (Fig. 2). The four $\beta$-strands in each subdomain form a $\beta$-sheet which participates in the formation of the substrate-transport path. In the primary sequence, PN1 and PN2 are tandemly inserted between TMs 1 and 2 of 
A

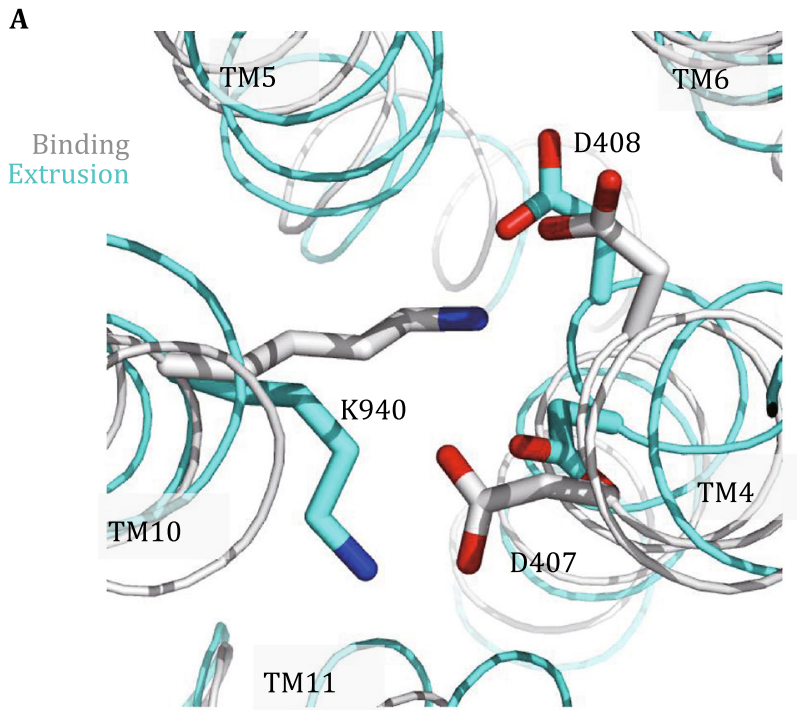

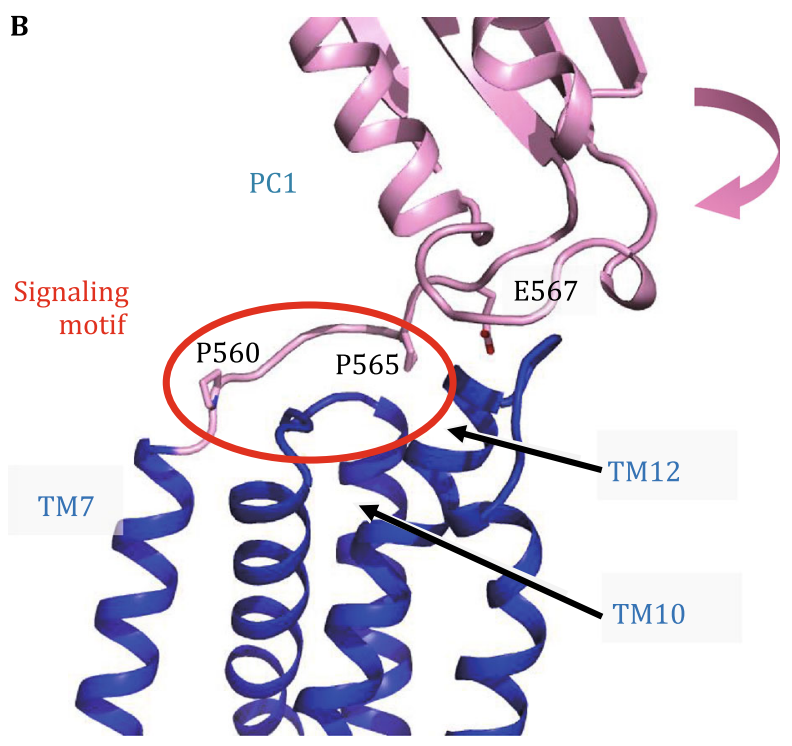

Fig. 3 Protonation switch in the TM domain. A The D407-D408-K940 cluster, viewed from the periplasm direction. The TM domains of the binding (gray) and extrusion (cyan) protomers are superimposed, with an overall RMSD of $1.3 \AA$ for $314 \mathrm{C} \alpha$-atom pairs. B Side view of the signaling motif between $\mathrm{C}_{\mathrm{TM}}$ (blue) and PC1 (pink). The substrate binding induced clockwise rotation of PC1 is postulated to further induce a clockwise rotation of TM10, which triggers protonation in TM4

$\mathrm{N}_{\mathrm{TM}}$, and PC1 and PC2 between TMs 7 and 8 of $\mathrm{C}_{\mathrm{TM}}$ (Fig. 2A; Fig. S1A). Furthermore, in the 3D structure, PN1 and PC2 pack together via a shared $\beta$-sheet, forming a rigid body (Eicher et al. 2014). So do PN2 and PC1, albeit significantly more flexible (when comparing different protomers). Thus, the porter domain in each AcrB protomer may be considered to be composed of two structural units, termed PN1-PC2 and PN2-PC1. The PN2-PC1 unit is observed to possess one of the major substrate binding sites (referred to as distal/deep binding site) (Murakami et al. 2006). The substrate entrance (also called proximal binding site) is located between the subdomains PC1 and PC2, laterally facing the periplasmic space. The substrate exit is located between the subdomains PN1 and PN2, connecting to a central exit chamber of the AcrB trimer. Thus, the entire substrate-transport path (including the entrance cleft, "major" binding site, and exit cleft) is formed between the two units, PN1-PC2 and PN2-PC1 (Li et al. 2015) (Fig. 2D). Importantly, exchanging the porter domains of AcrB and AcrD (an AcrB homolog) changes substrate specificity of the corresponding chimeric transporters (Elkins and Nikaido 2002). This result and other related studies firmly establish that the porter domain is the substrate-carrier domain of AcrB and determines the substrate specificity. In addition, the docking domain of an AcrB protomer is composed of two subdomains of similar folding, termed as DN (protruded from PN2) and DC (protruded from PC2), each of which also consists of two $\beta-\alpha-\beta$ repeats. Taken together, at the level of folding topology, both the porter domain and the docking domain of an AcrB protomer possess an internal pseudo twofold symmetry, which is an extension of the twofold symmetry of the TM domain (Fig. 2A).

In the homo-trimer, AcrB molecules form three layers parallel to the membrane plane (Fig. 2): (1) The three TM domains form a loosely packed ring structure located in the inner membrane. (2) The three porter domains form a tightly packed, membrane-proximal layer in the periplasm. A three-helix bundle is formed around the pseudo threefold-symmetry axis, with each protomer contributing one helix (termed gate helix, formerly called pore helix) from its PN1 subdomain. (3) The docking domains interlock with each other using a protruding $\beta$-hairpin (known as "peg") from each of the DN and DC subdomains, forming a rigid, threefold symmetrical, membrane distal layer. Folding homology between DN and DC subdomains further renders the layer an approximate sixfold symmetrical architecture, which may facilitate interactions of the docking layer with the homo-hexamer of the adapter AcrA in the assembled complex (Jeong et al. 2016). Furthermore, the dockingdomain trimer forms the central exit chamber (formerly called funnel), which extends into the cellular exterior via the TolC conduit (Fig. 1).

\section{PERISTALTIC MECHANISM OF ACRB}

Based on asymmetric tripartite structures of AcrB reported from multiple laboratories (Murakami et al. 2006; Seeger et al. 2006; Sennhauser et al. 2007), a 
peristaltic mechanism containing a three-step functional rotation has been proposed. In this functional cycle, each AcrB protomer successively runs through the access (A), binding (B), and extrusion (E) phases (also known as loose, tight, and open states). Furthermore, in a given crystal structure of the asymmetric trimer of AcrB, each protomer assumes one of the three different phases, with a counter-clockwise phase rotation (viewed from the TolC direction). Thus, although containing no physically rotating parts, the periplasmic porter domain cycles through three sequential phases, (1) capturing a lipophilic substrate from the peripheral periplasm-membrane interface, (2) transferring the substrate to the deep binding pocket, and (3) squeezing the substrate through the exit cleft into the central chamber.

In the functional cycle, the subdomains PN1, PN2, PC1, and PC2 change their packing conformations (Eicher et al. 2014). First, the entrance cleft between PC1 and PC2 opens laterally to the periplasm in the access phase, but gets partially closed in the binding phase. It is completely closed in the extrusion phase, with a relative rotation of $25^{\circ}$ between PC1 and PC2 during the binding-to-extrusion (B-E) transition. Secondly, the "major" binding site between PN2 and PC1 is of a more extended conformation in the access and binding phases, but contracts in the extrusion phase (with a relative rotation of $21^{\circ}$ ) essentially eliminating the substrate binding. Thirdly, the exit cleft between PN1 and PN2 is closed in the binding phase, but becomes open to the central chamber in the extrusion phase. This opening is partially caused by an $18^{\circ}$ relative rotation between PN1 and PN2 during the B-E transition, and partially by a gating movement of the gate helix from a neighboring promoter that is under the extrusion-to-access (E-A) transition.

This functional cycle requires external energy to drive, which is provided by the PMF-driven motors located in the three TM domains, in a fashion analogous to a three-cylinder car engine. As substrate transport and power generation are two functions physically separated (in the porter and TM domains, respectively), reliable communication and energy coupling between the two corresponding layers are essential for ensuring proper energy-transduction efficiency and thus high transport rate. Questions remaining to be addressed concerning the peristaltic mechanism include "How does substrate binding in the porter domain activate protonation of the TM domain?" and "How does protonation in the TM domain in turn drive the conformational change in the porter domain?" Finding answers to these questions will allow for a better understanding of the mechanistic details of RND transporters.

\section{PROTONATION IN ACRB TRANSPORTER}

As pointed out earlier, the protonation site in each EcAcrB protomer is composed of residues D407, D408 in TM4, and K940 in TM10. The asymmetric trimer structure of AcrB provides a number of clues as to how this putative protonation site may change its local conformation between distinct phases. The D407-D408 pair is located in the middle of the lipid bilayer and is insulated from bulk solvent. Because of the low dielectric constant of their environment, either of these two acidic residues may potentially be protonated. (See Ref. (Hanz et al. 2016) for an NMR study on the protonation of acidic residues in a TM helix.) On the other hand, by forming salt-bridge bonds, the nearby basic residue K940 may reduce the protonation ability (i.e., p $K_{\mathrm{a}}$ ) of the acidic pair. In both the access and binding protomers, the positive charge of the K940 side chain is located between the two negatively charged side chains of D407 and D408, preventing the acid residues from being protonated. The equilibrium conformation of such a deprotonated state (including its relative position to the membrane) will be considered as a reference for conformational changes upon protonation (see below). In contrast, in the extrusion protomer, the lysine side chain moves away from the acidic residues (Fig. 3A) and is stabilized by a H-bond formed with another functionally important residue T978 in TM11 (Takatsuka and Nikaido 2006). Therefore, in the extrusion phase $\mathrm{p} K_{\mathrm{a}}$ values of both aspartate residues increase, so that one of them (presumably of higher $\mathrm{p} K_{\mathrm{a}}$ ) will become protonated (Li et al. 2015). Once one of the two residues is protonated, the $\mathrm{p} K_{\mathrm{a}}$ of the other residue will decrease. Thus, it is unlikely that these two acidic residues are protonated simultaneously.

Next, we will discuss the sources and sinks of proton flux. Based on available structural evidence we put forward the following argument: Upon activation of the TM domain, a proton (carrying a positive charge) moves from the periplasmic side of the inner membrane, following the negative-inside electrostatic membrane potential $(\Delta \Psi)$, to the proton binding site at the D407D408 pair. As illustrated in the $1.9 \AA$ A -resolution crystal structure of AcrB (PDB ID: 4DX5), the proton-transfer wire, through which protons move, is likely to be formed by side chains of polar residues in TM helices as well as by membrane-embedded water molecules (Eicher et al. 2012). For examples, the highly conserved residues P906, G930, N941, R971, and G1010 (Fig. S1) are either directly involved in the formation of the proton-transfer wires, or facilitate binding of water molecules. Thus, the proton is likely to be transferred from a periplasmic pool to the D407-D408 pair via a 
mechanism similar to the Newton's cradle. Moreover, once the protomer passes the E-A transition state, the positive charge of the K940 side chain returns to the vicinity of the D470-D408 pair, and deprotonation occurs. The released proton will continue its crossmembrane journey, presumably releasing the rest of its PMF energy. As part of the proton release path, conserved R971 in TM11 is located on the cytosolic side of the D407-D408 pair. In particular, in the access and binding phases D407 is connected to R971 via a cluster of four water molecules, while in the extrusion phase this proton path is blocked by the two side chains of V411 and L944 which are conserved as hydrophobic residues in the AcrB-like family (Fig. S1). Taken together, depending on the protonation status, two distinct constellations of $\mathrm{H}$-bond networks are found inside the TM domain, thus establishing the pathway for proton loading and release.

A similar mechanism of substrate binding-induced protonation has been proposed for some transporters of the major facilitator superfamily (MFS) (Zhao et al. 2014). In the bacterial proton-dependent oligopeptide transporters (POTs), which import di- or tri-peptides by utilizing PMF energy, a cluster of conserved polar residues is present in the middle of the transmembrane region nearby the substrate binding site. This cluster typically includes one (or two) acidic residue(s), one (or two) positively charge residue(s), and other uncharged polar residues. For example, in the E. coli peptide- $\mathrm{H}^{+}$ symporter, YbgH, residues Q18, E21, Y22, and K118 form such a cluster. Upon binding a substrate peptide, the negatively charged carboxyl terminal group of the substrate attracts the positively charged side chain of K118 away from the clusters, thus promoting protonation of E21. Once E21 becomes protonated, $\Delta \Psi$ exerts an inward force to the transporter, triggering the outward-to-inward conformational change of the symporter. Regarding AcrB-like transporters, D408 is less conserved than D407 (Fig. S1A). Thus, many members from this transporter family have only one acidic residue as the protonation site. As suggested in the above YbgH case, a single acidic residue may function effectively as a protonation site, provided that some electronegative groups are present in its vicinity. In addition, as shown in Fig. S1, the presence of a basic residue in TM10 is not absolutely essential for alternating the protonation status of TM4. Nevertheless, removing a positive charge (e.g., K940 in Ec-AcrB) is likely accompanied by eliminating a negative charge (e.g., D408), and thus the protonation ability of the polar cluster is maintained. In such a case, the $\mathrm{p} K_{\mathrm{a}}$ value of the remaining buried acidic residue might be reduced by being approached by an H-bond donor. A similar deprotonation mechanism has been proposed recently for activation of class-A GPCRs (Zhang et al. 2014).

Results from several previous studies support our hypothesis. For instance, the D407-D408-K940 cluster of MexB (an AcrB homolog) has been shown to be very sensitive to point mutations. Any mutation that causes a charge change (e.g., D407N and D408N) results in loss of transport activity (Guan and Nakae 2001; Middlemiss and Poole 2004). In addition, a chemical modification assay using DCCD (dicyclohexylcarbodiimide) as a probe, which identifies protonatible acidic residue(s) in a hydrophobic environment, showed that D408 of EcAcrB can be protonated (Seeger et al. 2009). Recently, mutations in the proposed proton release path (residues V411, L944, and R971) have been shown to be lethal (Liu and Zhang 2017). These results support the notion that the D407-D408 pair forms part of the protontransfer wire for the PMF-driven mechanism.

\section{ENERGY COUPLING IN ACRB}

For Ec-AcrB, the relative movement K940 in TM10 away from the D407-D408 acidic pair in TM4 seems to represent the trigger for the protonation event occurring within the TM domain. In order to induce such a relative movement between TMs 4 and 10, the substrate binding signal must be transduced from the porter layer to the TM layer. The communication between the two layers is speculated to be long-distance in nature (Eicher et al. 2014; Yamaguchi et al. 2015), and possible mechanisms of signaling through direct physical transduction are currently ignored. By inspiring available crystal structures of AcrB, however, we proposed an alternative, interdomain contact-mediated mechanism for the signaling. We noticed that a short $\beta$-strand located inside an S-shaped loop connecting TM9 and TM10 forms a three H-bond, irregular, parallel $\beta$-sheet with another short $\beta$-strand in the loop connecting $\mathrm{C}_{\mathrm{TM}}$ to PC1 (Fig. 3B). We proposed that this $\beta$-sheet provides an information path for the substrate binding signal transduced from the porter layer to the TM layer, and therefore we term this sheet $\mathrm{PC} 1-\mathrm{C}_{\mathrm{TM}}$ signaling motif. Based on previously reported structures (Murakami et al. 2006; Seeger et al. 2006; Sennhauser et al. 2007), we proposed that this motif allows movement of PC1 upon substrate binding to be sensed by TM10, inducing a $\sim 12^{\circ}$ rotation of TM10 relative to the remaining part of $\mathrm{C}_{\mathrm{TM}}$ during the B-E transition. Furthermore, during the B-E transition, a similar though smaller movement is also observed for TM4. In particular, a short $\beta$-strand connecting TM3 and TM4 forms three H-bonds with a $\beta$ strand inside the $\mathrm{N}_{\mathrm{TM}}-\mathrm{PN} 1$ connecting loop, and we 
term this parallel $\beta$-sheet $\mathrm{PN} 1-\mathrm{N}_{\mathrm{TM}}$ signaling motif. Because of the presence of this motif, a movement of PN1 upon substrate binding may be sensed by TM4. Comparing the binding and extrusion phases reveals a $\sim 8^{\circ}$ rotation of the $\mathrm{N}$-terminal (periplasmic) half of TM4 relative to the $\mathrm{C}$-terminal (cytosolic) half, resulting in a helix bulge near the conserved G403 (of the $\mathrm{GX}_{3}$. $\mathrm{DX}_{6} \mathrm{E}$ motif) in the extrusion phase. As a consequence of this deformation, the side chain of D407 interacts with the backbone carbonyl oxygen of G403, presumably increasing the $\mathrm{p} K_{\mathrm{a}}$ value of D407. Interestingly, both the short $\beta$-strands in the $\mathrm{N}_{\mathrm{TM}}-\mathrm{PN} 1$ and $\mathrm{C}_{\mathrm{TM}}-\mathrm{PC} 1$ loops are flanked by two conserved proline residues (Fig. 3B; Fig. S1A). Together, the relative movement (with a net $14^{\circ}$ rotation) between TM10 and the C-terminal half of TM4 likely triggers protonation of the D407-D408 pair, using the abovementioned mechanism. Recently, it has been shown that mutations in the signaling motifs indeed reduce or abolish AcrB activity (Liu and Zhang 2017). In addition, although not directly located in the signaling motifs, several random point mutations in the porter-TM interface region were found to reduce the transport activity of MexB (e.g., S462F in the loop connecting TMs 5 and 6, E864K, V928M, and G1002D in the $\mathrm{N}$-terminal (periplasmic) regions of TMs 8, 10, and 12, respectively) (Middlemiss and Poole 2004). More importantly, PC1 is involved in substrate binding in both the access and binding phases. During the B-E transition, PC1 exhibits the largest movement, compared to other subdomains in the porter layer (Seeger et al. 2006). An AcrB inhibitor that binds between PN2 and PC1 in the B-state seems to block this movement of PC1 (Nakashima et al. 2013), thus breaking the communication between the porter and TM layers. Taken together, we proposed that the signaling motifs, especially that of $\mathrm{PC} 1-\mathrm{C}_{\mathrm{TM}}$, are essential for transducing substrate binding signal from the porter layer to TM layer.

Effective proton transfer across the membrane does not necessarily translate into efficient energy usage. For example, slippage in energy coupling might result in futile energy dissipation in theoretical models (Hill 1989). Thus, for an effective AcrB-like transporter, a mechanism of transducing the PMF energy into mechanical rearrangements in the porter layer likely exists. Similar to the case of PMF-driven MSF transporters (Zhang et al. 2015), the protonation at D407D408 pair is likely to exert an extra inward force to the $\mathrm{N}_{\mathrm{TM}}$-subdomain relative to the abovementioned reference state, causing a movement of the $\mathrm{N}_{\mathrm{TM}}$-subdomain towards the cytosolic direction. The strength of the electrostatic force is of an order of $5 \mathrm{pN}$, if the electrostatic field of the membrane potential is uniformly distributed across the $30-\AA \AA$ thickness of the membrane
(Zhang et al. 2015). A force of such a magnitude is sufficiently large to conduct many molecular biology events, such as moving of a motor protein along microtubules, separating of a dsDNA helix, or packaging a DNA molecule into a phage shell (Schnitzer et al. 2000; Cocco et al. 2001; Liu et al. 2014). Furthermore, embedding a proton-transfer wire into the membrane is equivalent to applying a focused membrane potential across the membrane, and thus the effective membrane thickness is reduced. Therefore, the extra electrostatic force upon protonation may be significantly larger than $5 \mathrm{pN}$. This extra electrostatic force may further be transduced from the TM domain to the porter domain via routes additional to the signaling motif, e.g., the structural connection between TM2 and PN2. Consequently, the corresponding subdomains in the porter domain are likely to move also in an inward direction. Such a movement will result in further conformational rearrangement necessary for the B-E transition, including a tilting of the gate helix in PN1 to block the exit cleft of the neighboring porter domain (Seeger et al. 2006).

As the TM domain moves out of the lipid bilayer, the hydrophobic mismatch between them will dramatically increase. This hydrophobic mismatch generates forces that partially balance the electrostatic force, such that no further out-membrane movement would be possible. Upon deprotonation, the energy stored in the form of a hydrophobic mismatch is released, returning the TM domain to its "reference" status (i.e., the access phase). In addition, the amphipathic helix $\alpha 6-7$, which is strategically located in the peripheral of the $\mathrm{C}_{\mathrm{TM}}$-subdomain, keeps $\mathrm{C}_{\mathrm{TM}}$ aligned with the membrane surface upon movement of the $\mathrm{N}_{\mathrm{TM}^{-}}$-subdomain towards the cytosol. Thus, a6-7 serves as a pivot point and may facilitate tilting of the TM domain as a whole. Disrupting this amphipathic helix (including the anchoring tyrosine residue) results in loss of the AcrB activity (Liu and Zhang 2017). Similar functional roles of amphipathic helices have been proposed earlier for GPCR activation (Zhang et al. 2014), conformational switching of MFS transporters (Jiang et al. 2013), and sensing of membrane tension in mechanosensitive channels (Zhang et al. 2016). Moreover, the loosely packed TM layer in the AcrB trimer (Fig. 2B) allows the three TM domains tilt independently in response of their individual protonation status. In the AcrB trimer, a differentially phased tilting process of the TM domains is likely to correlate with the packing rearrangement inside the porter layer.

In the high-resolution structures of asymmetric AcrB trimer (e.g., 4DX5), the TMs 1-4 in $\mathrm{N}_{\mathrm{TM}}$ form a groove facing the lipid bilayer. A detergent molecule was found 
to bind in this groove, suggesting that this site is a ligand- or inhibitor-binding site that may potentially interfere with the coupling between the TM domain and PN1. In the same crystal structure, a similar ligand binding site was also observed in the symmetric position in $\mathrm{C}_{\mathrm{TM}}$. Interestingly, in a recently reported crystal structure of human cholesterol transporter, NPC1 (PDB ID: 5I31, a member of the RND superfamily), a sterolsensing domain (SSD) is formed by helices TMs 3-5 (equivalent to TMs 2-4 in AcrB). Both genetic and structural analyses suggest that this region is important for NPC1 function. The head group of the cholesterol molecule bound to the SSD may interfere with the putative signaling $\beta$-sheet between the TM domain and the exo-membrane domain. Therefore, the signaling motifs that we proposed here for Ec-AcrB may indeed be a general feature for RND transporters.

We would like to further emphasize that the putative protonation-induced movement requires the presence of a cross-membrane electrochemical potential of protons, particularly the negative-inside $\Delta \Psi$. Such precondition was not taken into account in most in vitro experiments reported so far, neither for AcrB functional nor for structural studies. Even in molecular dynamic simulations, $\Delta \Psi$ was usually not included as part of the environmental condition (Schulz et al. 2015). The absence of membrane potential may explain why the observed transport rate of AcrB was slow (approx. one substrate molecule per minute) in a proteoliposomebased transport assay (Nikaido and Takatsuka 2009), in contrast to a much higher efficiency during in vitro transport (e.g., $\sim 100 / \mathrm{s}$ for substrate ampicillin) (Li et al. 2015).

\section{FREE-ENERGY LANDSCAPE}

Functional separation of substrate efflux and proton influx in distinct domains is a characteristic feature of AcrB-like RND transporters (Yamaguchi et al. 2015). Because of this separation, Jardetzky's classical alternating access model (Jardetzky 1966) appears to be not directly applicable to RND transporters. However, the AcrB-like transporters maintain an important feature of most transporters, i.e., switching back and forth between conformations of high and low affinities towards a given substrate. In this sense, the two structural units of the AcrB porter domain, namely PN1-PC2 and PN2-PC1, may be analogous to the $\mathrm{N}-$ and C-transmembrane domain in an MFS transporter. Whereas the relative movement between the two domains in MFS results in an in-membrane conformational change in agreement to the Jardetzky model, the relative movement between PN1-PC2 and PN2-PC1 results in a conceptually similar conformation alternation, though in the periplasm. A major difference between these two types of transporters is the energy coupling mechanism utilized. For instance, in the case of an MFS antiporter, a direct competition between the substrate and the driving substance, proton or $\mathrm{Na}^{+}$, is postulated (Zhang et al. 2015). In contrast, the energy coupling mechanism appears to be more complicated in the case of AcrB-like transporters (Eicher et al. 2014). As a tool to dissect the energy coupling mechanism of AcrB, we introduce its free-energy landscape plot, as shown in Fig. 4.

By definition, substrates of AcrB are compounds that are able (1) to bind to the access state and (2) to induce subsequent transitions. These rather general criteria allow AcrB to accommodate a broad substrate specificity, e.g., that observed in multidrug resistance (Yamaguchi et al. 2015). As discussed earlier, the second criterion is equivalent to the ability of the substrate to induce a rotation of the PC1 subdomain, thus triggering protonation in the TM domain. However, for a substrate to meet the first requirement, the loadingsite dissociation constant $K_{\mathrm{d}, \mathrm{L}}$ should be smaller than the releasing-site dissociation constant $K_{\mathrm{d}, \mathrm{R}}$, so that the substrate can be captured from a low-concentration environment and be released into a high-concentration environment. Such a difference in substrate binding affinity between two conformations of a transporter is associated with the free-energy term, previously named differential binding energy, i.e., $\Delta G_{\mathrm{D}}$ ( $\equiv$ $R T \cdot \ln \left(K_{\mathrm{d}, \mathrm{R}} / K_{\mathrm{d}, \mathrm{L}}\right)>0$ ) (Zhang et al. 2015) (see Box 1 ). Without an external energy input, the transporter would not proceed sustainably to transform from a high-affinity state into a lower-affinity state. In order for the functional cycle of substrate export to proceed, the positive $\Delta G_{\mathrm{D}}$ must be compensated by the driving energy, e.g., PMF. In the three-step functional cycle, $\Delta G_{\mathrm{D}}$ for the substrate may be further divided into two terms associated with the A-B and B-E transitions (Fig. 4B). Furthermore, a good substrate usually reduces the height of the energy barrier of the transition state(s) (indicated as green horizontal lines in Fig. 4B), but poor substrates or inhibitors lack this ability. Such a reduction can be achieved through stronger binding of the substrate to the transporter at the transition state relative to the "ground" state. This mechanism of reducing the transition-state barrier is likely to also contribute to the substrate specificity observed for transporters (Zhang and Han 2016).

The three transitions between successive phases are associated with conformational energy changes, termed 
A

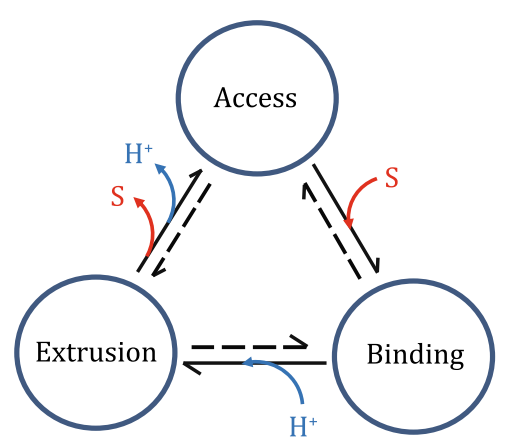

C

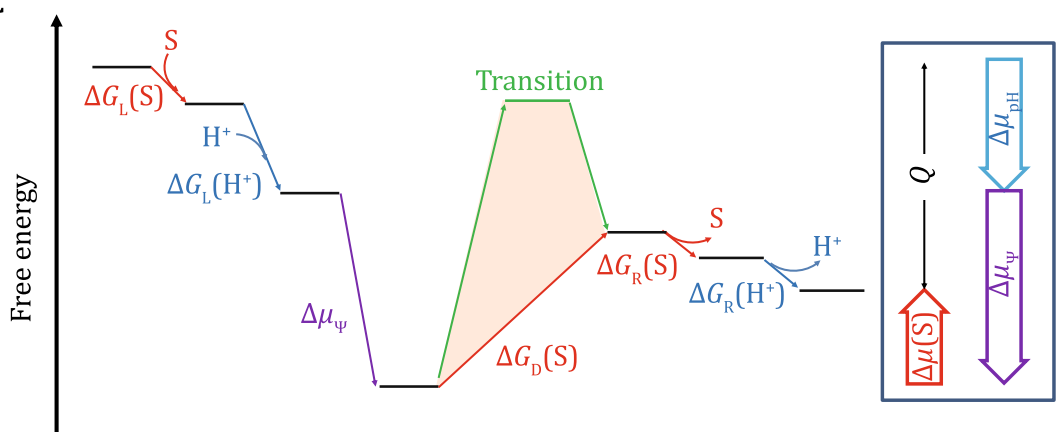

B

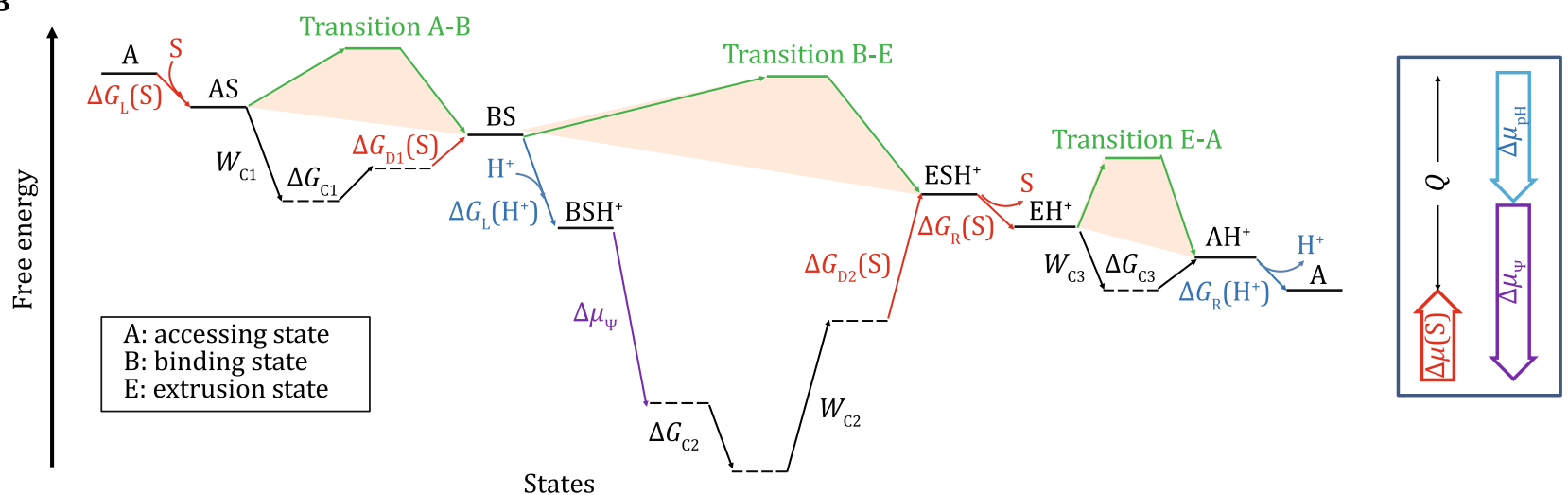

Fig. 4 Free-energy landscape of the AcrB protomer. A King-Altman plot of the three-state functional cycle of AcrB. The dominant directions of the reactions are shown in solid half arrows. B Energy landscape of the AcrB protomer. The plot must satisfy the First and Second Laws of thermodynamics (right-side Box). The vertical axis represents the Gibbs free energy. Horizontal lines represent states, and the horizontal axis is essentially an expansion of the King-Altman plot. Thus, tilted lines represent transitions between states. Transitions associated with the proton binding are indicated in blue, those with the substrate in red, and those with $\Delta \Psi$ in purple. For simplicity, the electrostatic energy is shown as one package, although it could be separated into two parts associated with proton loading and releasing. In addition, kinetic terms are shown in green. Subscripts "L", " $\mathrm{R}$ ", and " $\mathrm{D}$ " stand for energy terms associated with loading, releasing, and differential binding, respectively. The intrinsic conformational energy terms, $\Delta G_{\mathrm{C} 1 / 2 / 3}$, and energy terms for cooperativity work, $W_{\mathrm{C} 1 / 2 / 3}$, are discussed in the main text. In principle, since the transport process cycles, the choice of the starting point is arbitrary. Therefore, the starting and ending states are considered identical, only differing by the amount of heat $(Q)$ dissipated during one transport cycle. Thus, the end state must be located below the starting state. Neighboring states may be tightly coupled energetically. In such a case, their sequential order would be arbitrary. Locally, any transition of positive $G$ is likely to be driven by a coupled transition of a negative $G$ (also see Box 1). C Energy landscape plot of the AcrB trimer. Because of cooperativity between the three protomers, the intrinsic conformational energy terms, $\Delta G_{\mathrm{C} 1 / 2 / 3}$, and energy terms for cooperativity work, $W_{\mathrm{C} 1 / 2 / 3}$, shown in $\mathbf{B}$ cancel each other out. Thus, the plot is simplified. Only one-third of the functional cycle is shown, and the remaining two-thirds are its precise repeats

$\Delta G_{\mathrm{C} 1}, \Delta G_{\mathrm{C} 2}$, and $\Delta G_{\mathrm{C} 3}$, which are intrinsic property of the transporter and are independent of the substrate binding. As a hypothetical example, as shown in Fig. 4B, the $\mathrm{B}$ (binding) state is of higher conformational energy than the A (access) state $\left(\Delta G_{\mathrm{C} 1}>0\right)$. The positive $\Delta G_{\mathrm{C} 1}$ suggests that in the absence of external energy, the $A-B$ transition is thermodynamically unfavorable. There are many factors that may contribute to these differential conformational energy terms. For instance, the abovementioned hydrophobic mismatch may be considered as a contributor of the conformational energy in the extrusion phase, thus affecting both $\Delta G_{\mathrm{C} 2}$ and $\Delta G_{\mathrm{C} 3}$. Since the promoter must return to its initial state after a complete functional cycle, the sum of these energy terms associated with the three conformational changes should be zero:

$\Delta G_{\mathrm{C} 1}+\Delta G_{\mathrm{C} 2}+\Delta G_{\mathrm{C} 3}=0$.

Conceptually, if the protomers were isolated from the trimer, such conformational energy terms could be determined by measuring its population distribution in different states at equilibrium. Due to the functional coupling between the protomers, however, these energy terms $\left(\Delta \mathrm{G}_{\mathrm{C} 1}, \Delta G_{\mathrm{C} 2}\right.$, and $\left.\Delta G_{\mathrm{C} 3}\right)$ may not be determined experimentally for an AcrB-like transporter.

One characteristic feature of the AcrB trimer is the absolute requirement of functional cooperation between the three protomers (Eicher et al. 2014). It has been 
Box 1 Definition of the electrochemical potential terms

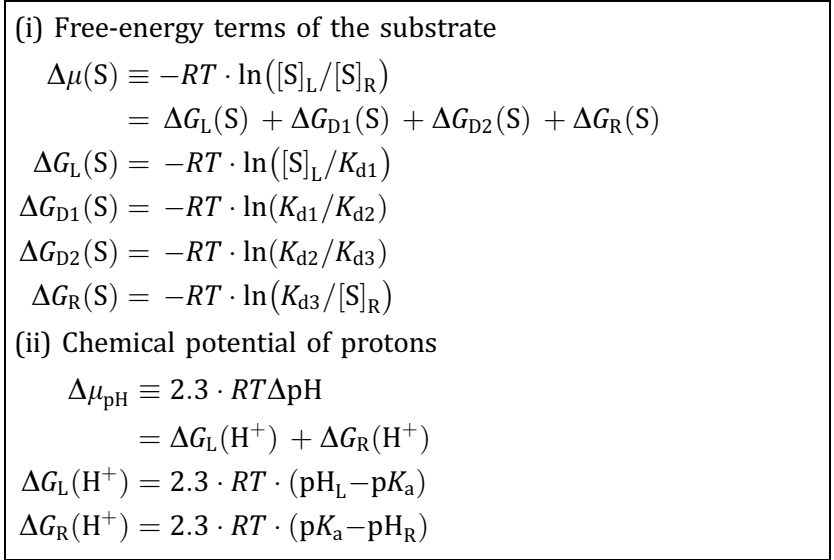

shown that, in a genetically fused AcrB trimer, deactivating one single protomer abolishes the activity of the entire trimer complex (Takatsuka and Nikaido 2009). Furthermore, not all external energy (i.e., $\Delta \mu_{\mathrm{pH}}+F \Delta \Psi$, where $F$ is the Faraday constant and $\Delta \mu_{\mathrm{pH}}$ is explained in Box 1) is released as one package to drive the B-E transition. Part of the input energy $\left(W_{\mathrm{C} 2}\right)$ may be considered as output work for "cooperativity." In other words, input energy generated by proton influx in one protomer is partially used to allow the other two protomers to overcome their transition barriers as well as thermodynamically unfavorable $\Delta G_{\mathrm{C}}$ terms. Like $\Delta G_{\mathrm{C} 1 / 2 / 3}$ terms, the energy terms associated with cooperativity is also in balance:

$W_{\mathrm{C} 1}+W_{\mathrm{C} 2}+W_{\mathrm{C} 3}=0$.

For a hypothetical, isolated, monomeric transporter, relative free-energy levels (and the associated rate constants) of each state together would determine the population probability among different states. This distribution in turn would affect the overall transport rate of the transporter (Hill 1989). The rates of three transitions, A-B, B-E, and E-A, would be different. However, because of the cooperativity within one AcrB trimer, each protomer is present in one of the three major states. Therefore, the population probability of each major state is virtually $1 / 3$. This imposes a strong restriction both on the free-energy landscape and on the kinetics of the transitions between successive states (see Box 2). By automatically adjusting energy terms of cooperative work $\left(W_{\mathrm{C} 1}, W_{\mathrm{C} 2}\right.$, and $\left.W_{\mathrm{C} 3}\right)$, rates at the three transitions are maintained to be the same. For instance, if the substrate concentration at the loading site increases, the pseudo first-order rate constant $\left([\mathrm{S}]_{\mathrm{L}} k_{\mathrm{on}}^{0}\right)$ will increase (Hill 1989). This increased rate constant would shift the population probability from the empty access state (A) to the substrate-bound access state (AS)

\section{Box 2 Cooperativity}

Assumption: The cooperative complex contains $N$ copies of identical protomers, and each of them is in a distinct transition-state (or phase) of the functional cycle

(i) Energy conservation

$$
\sum_{i}^{N} W_{\mathrm{C} i}=0
$$

where $W_{\mathrm{C} i}$ is the cooperative work that is output by the protomer in the $i$-th step. A negative $W_{\mathrm{C} i}$ would indicate that the $i$-th step absorbs energy from the cooperative complex

(ii) Arrhenius theorem

$$
k_{i} \mathrm{e}^{-W c i / R T}=k_{j} \mathrm{e}^{-W c j / R T}
$$

where $k_{i}$ is the (pseudo) first-order rate constant of the $i$-th step in a hypothetical non-cooperative protomer

(iii) Cooperative work

$$
\begin{aligned}
& W_{C i}=\frac{-R T}{N} \ln \frac{\prod_{j}^{N} k_{j}}{k_{i}^{N}}=R T \ln \frac{k_{i}}{k_{\mathrm{c}}} \\
& k_{\mathrm{c}}=\left(\prod_{j}^{N} k_{j}\right)^{\frac{1}{N}}
\end{aligned}
$$

where $k_{\mathrm{c}}$ is the pseudo first-order rate constant of the cooperative complex. For instance, for the AcrB trimer, $k_{\mathrm{C}}=$ $\left(k_{1} \cdot k_{2} \cdot k_{3}\right)^{1 / 3}$

and would make the A-B transition run faster. Thus, the external energy required for this transition decreases (i.e., $W_{\mathrm{C} 1}$ becomes less negative) in order to maintain all three transitions at the same rate. In contrast, in the absence of any substrate at the loading site, the A-B transition as well as the functional cycle stops. In short, the input PMF energy will be distributed dynamically among the three cooperative promoters of the transporter trimer, depending on the types and concentrations of substrate molecules associated with each protomer. For a given type of substrate at a fixed concentration gradient, the cooperativity works $W_{\mathrm{C} 1}, W_{\mathrm{C} 2}$, and $W_{\mathrm{C} 3}$ from all three protomers cancel each other out at any given moment. Furthermore, according to the Arrhenius theorem, the pseudo first-order rate constant of a cooperative transporter complex will change by a factor of $\exp \left(-W_{\mathrm{C} i} / R T\right)$ (where $\left.i=1,2,3\right)$, relative to the hypothetical rate of an isolated monomeric transporter (Box 2). In addition, $\Delta G_{\mathrm{C} 1}, \Delta G_{\mathrm{C} 2}$, and $\Delta G_{\mathrm{C} 3}$ from the three protomers balance each other, albeit they are intrinsic properties of the transporter. Thus, the energy landscape plot of a cooperative AcrB trimer can be presented in a simplified manner, as shown in Fig. 4C. A key feature of this simplified plot is that PMF, including energy terms associated with both $\Delta \mathrm{pH}$ and $\Delta \Psi$, remains to be utilized to compensate the positive $\Delta G_{\mathrm{D}}(\mathrm{S})$ in thermodynamic terms and to overcome the transition state energy barrier in kinetic terms. 


\section{PERSPECTIVE}

The United Nations General Assembly recently unanimously approved a declaration committing nations to a more active battle against threat of microbial resistance to existing drugs, which is one of the most severe and rapidly growing threats to contemporary public health. Massive intergovernmental efforts will be required to achieve such an ambitious undertaking. Since bacteria develop drug resistance naturally in an environment containing antibiotics, studies on the mechanism of multidrug resistance, including those of AcrB-like transporters, are an essential part of our fight against multidrug resistance (Li et al. 2015). Based on the progress in structural and functional studies on AcrBlike transporters in the last 15 years, a new energy coupling mechanism is proposed in the current review for this major type of multidrug-resistance transporters. If verified, this mechanism is expected to permit us to de-couple between their substrate binding and energy converting. Experimental approaches stemmed from this mechanistic hypothesis may thus open new avenues for developing novel inhibitors to attack the multidrug resistance mediated by AcrB-like transporters.

\section{Abbreviations \\ PMF Proton motive force \\ RND Resistance-nodulation-cell division (transporter) \\ TM Transmembrane (helix)}

Acknowledgement We thank Dr. Torsten Juelich for linguistic assistance during the preparation of this manuscript. This work was supported by the Ministry of Science and Technology (China) (2015CB910104 to XCZ) and National Natural Science Foundation of China (31470745 to XCZ).

\section{Compliance with Ethical Standards}

Conflict of interest Xuejun C. Zhang, Min Liu, and Lei Han declare that they have no conflict of interest.

Human and animal rights and informed consent This article does not contain any studies with human or animal subjects performed by any of the authors.

Open Access This article is distributed under the terms of the Creative Commons Attribution 4.0 International License (http:// creativecommons.org/licenses/by/4.0/), which permits unrestricted use, distribution, and reproduction in any medium, provided you give appropriate credit to the original author(s) and the source, provide a link to the Creative Commons license, and indicate if changes were made.

\section{References}

Berman HM, Westbrook J, Feng Z, Gilliland G, Bhat TN, Weissig H, Shindyalov IN, Bourne PE (2000) The protein data bank. Nucleic Acids Res 28:235-242

Cocco S, Monasson R, Marko JF (2001) Force and kinetic barriers to unzipping of the DNA double helix. Proc Natl Acad Sci USA 98:8608-8613

Du D, Wang Z, James NR, Voss JE, Klimont E, Ohene-Agyei T, Venter H, Chiu W, Luisi BF (2014) Structure of the AcrAB-TolC multidrug efflux pump. Nature 509:512-515

Eicher T, Cha HJ, Seeger MA, Brandstatter L, El-Delik J, Bohnert JA, Kern WV, Verrey F, Grutter MG, Diederichs K, Pos KM (2012) Transport of drugs by the multidrug transporter AcrB involves an access and a deep binding pocket that are separated by a switch-loop. Proc Natl Acad Sci USA 109:5687-5692

Eicher T, Seeger MA, Anselmi C, Zhou W, Brandstatter L, Verrey F, Diederichs K, Faraldo-Gomez JD, Pos KM (2014) Coupling of remote alternating-access transport mechanisms for protons and substrates in the multidrug efflux pump AcrB. Elife. doi:10.7554/eLife.03145

Elkins CA, Nikaido H (2002) Substrate specificity of the RND-type multidrug efflux pumps AcrB and AcrD of Escherichia coli is determined predominantly by two large periplasmic loops. J Bacteriol 184:6490-6498

Gong X, Qian H, Zhou X, Wu J, Wan T, Cao P, Huang W, Zhao X, Wang X, Wang P, Shi Y, Gao GF, Zhou Q, Yan N (2016) Structural insights into the Niemann-Pick C1 (NPC1)-mediated cholesterol transfer and ebola infection. Cell 165:1467-1478

Guan L, Nakae T (2001) Identification of essential charged residues in transmembrane segments of the multidrug transporter MexB of Pseudomonas aeruginosa. J Bacteriol 183:1734-1739

Hanz SZ, Shu NS, Qian J, Christman N, Kranz P, An M, Grewer C, Qiang W (2016) Protonation-driven membrane insertion of a pH-low insertion peptide. Angew Chem Int Ed Engl 55:12376-12381

Higgins CF (2007) Multiple molecular mechanisms for multidrug resistance transporters. Nature 446:749-757

Hill TL (1989) Free energy transduction and biochemical cycle kinetics. Springer, New York

Jardetzky O (1966) Simple allosteric model for membrane pumps. Nature 211:969-970

Jeong H, Kim JS, Song S, Shigematsu H, Yokoyama T, Hyun J, Ha NC (2016) Pseudoatomic structure of the tripartite multidrug efflux pump AcrAB-TolC reveals the intermeshing cogwheellike interaction between AcrA and TolC. Structure 24:272-276

Jiang D, Zhao Y, Wang X, Fan J, Heng J, Liu X, Feng W, Kang X, Huang B, Liu J, Zhang XC (2013) Structure of the YajR transporter suggests a transport mechanism based on the conserved motif A. Proc Natl Acad Sci USA 110:14664-14669

Li XZ, Plesiat P, Nikaido H (2015) The challenge of efflux-mediated antibiotic resistance in Gram-negative bacteria. Clin Microbiol Rev 28:337-418

Liu M, Zhang XC (2017) Energy-coupling mechanism of the multidrug resistance transporter AcrB: evidence for membrane potential-driving hypothesis through mutagenic analysis. Protein Cell 8(8):623-627

Liu S, Chistol G, Hetherington CL, Tafoya S, Aathavan K, Schnitzbauer J, Grimes S, Jardine PJ, Bustamante C (2014) A viral packaging motor varies its DNA rotation and step size to preserve subunit coordination as the capsid fills. Cell 157:702-713 
Middlemiss JK, Poole K (2004) Differential impact of MexB mutations on substrate selectivity of the MexAB-OprM multidrug efflux pump of Pseudomonas aeruginosa. J Bacteriol 186:1258-1269

Murakami S, Nakashima R, Yamashita E, Yamaguchi A (2002) Crystal structure of bacterial multidrug efflux transporter AcrB. Nature 419:587-593

Murakami S, Nakashima R, Yamashita E, Matsumoto T, Yamaguchi A (2006) Crystal structures of a multidrug transporter reveal a functionally rotating mechanism. Nature 443:173-179

Nakashima R, Sakurai K, Yamasaki S, Hayashi K, Nagata C, Hoshino K, Onodera Y, Nishino K, Yamaguchi A (2013) Structural basis for the inhibition of bacterial multidrug exporters. Nature 500:102-106

Nikaido H, Takatsuka Y (2009) Mechanisms of RND multidrug efflux pumps. Biochim Biophys Acta 1794:769-781

Perrin E, Fondi M, Papaleo MC, Maida I, Buroni S, Pasca MR, Riccardi G, Fani R (2010) Exploring the HME and HAE1 efflux systems in the genus Burkholderia. BMC Evol Biol 10:164

Schnitzer MJ, Visscher K, Block SM (2000) Force production by single kinesin motors. Nat Cell Biol 2:718-723

Schulz R, Vargiu AV, Ruggerone P, Kleinekathofer U (2015) Computational study of correlated domain motions in the AcrB efflux transporter. Biomed Res Int 2015:487298

Seeger MA, Schiefner A, Eicher T, Verrey F, Diederichs K, Pos KM (2006) Structural asymmetry of AcrB trimer suggests a peristaltic pump mechanism. Science 313:1295-1298

Seeger MA, von Ballmoos C, Verrey F, Pos KM (2009) Crucial role of Asp408 in the proton translocation pathway of multidrug transporter AcrB: evidence from site-directed mutagenesis and carbodiimide labeling. Biochemistry 48:5801-5812
Sennhauser G, Amstutz P, Briand C, Storchenegger 0, Grutter MG (2007) Drug export pathway of multidrug exporter AcrB revealed by DARPin inhibitors. PLoS Biol 5:e7

Sennhauser G, Bukowska MA, Briand C, Grutter MG (2009) Crystal structure of the multidrug exporter MexB from Pseudomonas aeruginosa. J Mol Biol 389:134-145

Takatsuka Y, Nikaido H (2006) Threonine-978 in the transmembrane segment of the multidrug efflux pump AcrB of Escherichia coli is crucial for drug transport as a probable component of the proton relay network. J Bacteriol 188:7284-7289

Takatsuka Y, Nikaido H (2009) Covalently linked trimer of the AcrB multidrug efflux pump provides support for the functional rotating mechanism. J Bacteriol 191:1729-1737

Yamaguchi A, Nakashima R, Sakurai K (2015) Structural basis of RND-type multidrug exporters. Front Microbiol 6:327

Zhang XC, Han L (2016) Uniporter substrate binding and transport: reformulating mechanistic questions. Biophys Rep 2:45-54

Zhang XC, Cao C, Zhou Y, Zhao Y (2014) Proton transfer-mediated GPCR activation. Protein Cell 6:12-17

Zhang XC, Zhao Y, Heng J, Jiang D (2015) Energy coupling mechanisms of MFS transporters. Protein Sci 24:1560-1579

Zhang XC, Liu Z, Li J (2016) From membrane tension to channel gating: a principal energy transfer mechanism for mechanosensitive channels. Protein Sci. doi:10.1002/pro.3017

Zhao Y, Mao G, Liu M, Zhang L, Wang X, Zhang XC (2014) Crystal structure of the E. coli peptide transporter $\mathrm{YbgH}$. Structure 22:1152-1160 